\title{
On Controls in Ancient Microbiome Studies, and Microbial Resilience in Ancient Samples
}

\author{
Tasha M. Santiago-Rodriguez ${ }^{1, *}$ and Gary A. Toranzos ${ }^{2}$ \\ 1 Diversigen Inc., Houston, TX 77046, USA \\ 2 Environmental Microbiology Laboratory, Biology Department, University of Puerto Rico, \\ San Juan, PR 00932, USA; gary.toranzos@upr.edu \\ * Correspondence: tsantiagoro@gmail.com
}

Received: 14 August 2018; Accepted: 21 September 2018; Published: 27 September 2018

\begin{abstract}
In the following comment, we reply to Eisenhofer and Weyrich's letter "Proper authentication of ancient DNA is still essential" responding to the article "Gut Microbiome and Putative Resistome of Inca and Italian Nobility Mummies" by Santiago-Rodriguez et al. One of the concerns raised was the possibility that the patterns noted in the gut microbiome of pre-Inca/Inca and Italian nobility mummies were due to contamination of the blank control. When examining the blank controls and filtering the operational taxonomic units (OTUs) present in the blank controls, and further performing in-silico contamination analyses, we noticed very similar patterns as those previously reported. We also discuss controls in ancient microbiome studies, and aspects of microbial resilience in ancient samples.
\end{abstract}

Keywords: blank controls; microbiome; mummies; preservation

\section{Controls in Ancient Microbiome Studies}

Several points should be addressed regarding Eisenhofer and Weyrich's letter responding to the article "Gut Microbiome and Putative Resistome of Inca and Italian Nobility Mummies" by Santiago-Rodriguez et al. published last year in the journal Genes [1,2]. Firstly, data presented in Figure $1 \mathrm{~A}$ are not at the species level, as claimed by Eisenhofer and Weyrich [1]. To clarify and to put results into perspective, 14 genera have been classified within the Sphingomonadales order alone, and a number of different operational taxonomic units (OTUs) can be classified within this order; therefore, OTUs classified as Sphingomonadales in the mummified guts may not necessarily represent those identified in the blank control. As stated in our study, the majority of the OTUs could not be classified at higher taxonomical levels. The limitations of being unable to classify data at the species level are intrinsic limitations within the microbiome field. One possible reason is that only one $16 \mathrm{~S}$ region was sequenced in the study (V4 region), and stringent parameters were applied, possibly limiting our ability to resolve species-level classification. Also, it has been shown in modern and ancient microbiome studies that the taxonomic classifier can influence results [3-5].

Secondly, several of the bacterial orders highlighted in Figure 1A by Eisenhofer and Weyrich are bacteria that can be found in several different environments including, and not surprisingly, the human gut. Sphingomonas spp., for example, have been recognized as essential in maintaining human gut homeostasis [6]. Other bacteria that are present in potentially contaminating environments (i.e., soil, skin and built environments) are also members of the human gut microbiome (Table 1). Relative abundance data are shown in Table 1 as percentages. While some of these bacterial orders are present in higher relative abundances in certain individuals (i.e., Bacillales in mummy FI3, Clostridiales in mummies FI9 and FI12, and Sphingomonadales in mummies NASD3 and NASD14), results show that most of the bacterial orders of concern to Eisenhofer and Weyrich are present in low relative 
abundances, representing $<1.0 \%$ of the microbiome in most of the mummies (Table 1). Notably, taxonomic data are normally presented as relative abundances, and percentages often represent numbers that are relative to the total number of reads in a sample. In addition, as noted by Eisenhofer and Weyrich, blank controls showed signals for specific bacterial OTUs. As microbiologists assessing the composition of such samples, it is our responsibility to report the OTUs present in the blank control. Various laboratories conducting ancient microbiome research have reported the presence of sequences in their blank controls as well $[7,8]$; thus, it is evident that many blank controls have inherent bacterial contamination [9]. In addition, detection of OTUs in the blank control can be affected by how these samples were processed. Specifically, if the blank control was processed along with the samples of interest, cross-contamination can occur. The extent of the cross-contamination can depend on how the samples were handled. On the other hand, if the blank control is processed separately from the rest of the samples, cross-contamination is less likely to occur [10]. Ideally, blank controls should be processed along with the samples of interest to provide an idea of the extent of the cross-contamination. The microbiome research community is currently discussing if OTUs present in a blank control should be removed from the sample of interest. One main reason is that a number of these OTUs present in a blank control may be part of the autochthonous microbiome of the sample of interest [11]; thus, filtering OTUs may not necessarily be a standard in microbiome research in all cases.

Table 1. Abundances (\%) of selected bacterial taxa at the order level in the pre-Inca/Inca (FI3, FI9, and FI12) and Italian nobility mummies (NASD3, NASD14, NASD22, NASD27, and NASD29), and the blank control. The percentages are relative to the total number of reads in each sample.

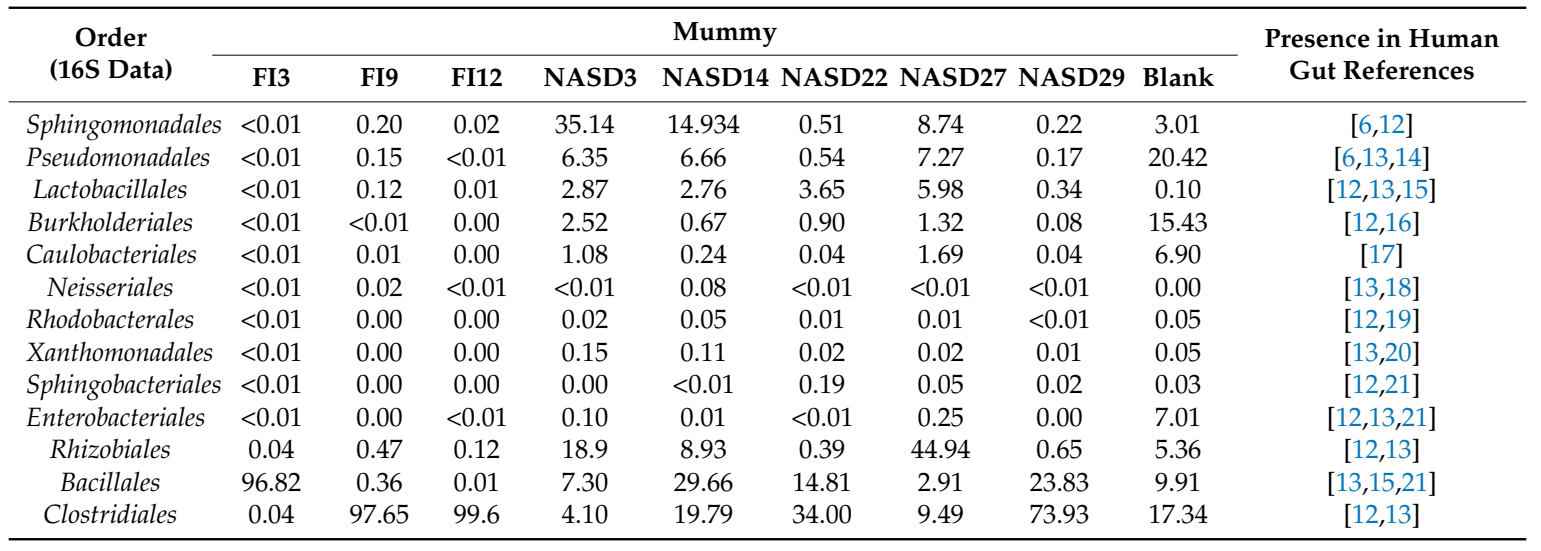

Thirdly, if the blank control microbiome was to significantly alter the microbiome composition, all the samples would have reflected the same level of contamination. This has been our experience when working with other low-biomass microbiomes, in which blank controls can overwhelm the resident microbiota of the sample of interest at very similar proportions across the samples, and sequences need to be discarded. The reasons for the differences in the relative abundances of the "contaminant" sequences (as stated by Eisenhofer and Weyrich) across the individuals remain unknown. However, in addition to these samples undergoing changes in water content, temperature, oxygen, and $\mathrm{pH}$ levels (all of which are known to affect the membership and function of microbial communities) [22-25], all of the mummies exhibited differences in terms of age, sex, diet, and comorbidities, factors that are also known to shape the microbiome [11].

To assess if the OTUs present in the blank control affected the interpretation of the previous results, we ran the biom summarize-table script to assess the total number of counts or sequences per sample. The pre-Inca/Inca mummies showed 78,903 (mummy FI3); 24,956 (mummy FI9); and 56,971 (mummy FI12) counts or sequences per sample. When running the script for the Italian nobility mummies, 32,086 (mummy NASD3); 44,255 (mummy NASD14); 60,266 (mummy NASD22); 24,633 (mummy NASD27); and 101,517 (mummy NASD29) counts or sequences per sample were identified. The blank control showed 3824 counts or sequences. To address if the 3824 counts or sequences identified in the 
blank control could affect the separation of the $16 \mathrm{~S}$ data based on culture, we filtered the OTUs present in the blank control from the biom table of the mummies using the script filter_otus_from_otu_table.py. The total number of counts or sequences pre- and post-filtering are reported in Table 2 . The number of OTUs in the blank control is also shown in Table 2. As stated, the number of OTUs in a blank control can differ depending on how it was processed. We also added the OTUs identified in the blank control to the filtered OTU table to mimic in-silico contamination. Briefly, OTU tables from the mummies and the blank control were merged using the merge_otu_tables.py script available in the Quantitative Insights Into Microbial Ecology (QIIME) package [26]. We then performed diversity and taxonomical analyses using the core_diversity_analyses.py script in QIIME. Results of the filtered samples virtually demonstrate similar dynamics as shown in our previous paper, which included non-filtered samples. Pre-Inca/Inca mummies showed lower observed Operational Taxonomic Units (OTUs) (Figure 1A) and evenness (Shannon diversity index) (Figure 1B) values compared to the Italian nobility mummies. In-silico contamination of the mummies OTU tables showed no significant differences in the alpha-diversity when looking at both observed OTUs (Figure 1C) and evenness (Shannon diversity index) (Figure 1D). Beta diversities were visualized as Principal Coordinate Analyses (PCOA) plots and showed a statistically significant separation of the data based on culture (Bray-Curtis index; the P-value, R-squared, and F-statistic values are shown (PERMANOVA test); Figure 2A). Hierarchical cluster plots of the beta-diversity (Bray-Curtis index) also showed a clear separation of the data based on culture (Figure 2B). Beta-diversity (Bray-Curtis index), visualized as PCoA plots, of the in-silico contaminated mummies OTU tables showed a separation of the data based on culture (The P-value, R-squared, and F-statistic values are shown (PERMANOVA test); Figure 2C). Hierarchical cluster plots showed that one of the Italian nobility mummies and one the Inca mummies did not cluster with the corresponding culture. This may suggest that in-silico contamination of the mummies OTU tables may affect, to some extent, the beta-diversity of certain samples. Heatmaps of the relative abundances of bacterial OTUs at the order level showed a separation of the data based on culture, except for the pre-Inca mummy (FI9), but closely resembled the Inca mummies (Figure 3A). We also performed the analysis at the genus level and noticed a separation of the data based on culture (Figure 3B). Heatmaps of the relative abundance of bacterial OTUs at the order level of the in-silico contaminated mummies OTU tables showed that two of the Italian nobility mummies microbiomes did not cluster with the rest of the Italian nobility microbiomes (Figure 3C). Similar outcomes were observed at the genus level (Figure 3D). This may suggest that in-silico contamination of the mummies OTU tables may affect, to some extent, clustering of the taxonomic data. These results support our previous study and suggest that OTUs present in a blank control may not necessarily affect the structure of a microbiome of interest.

Table 2. Sequence counts per sample prior and after filtering of operational taxonomic units (OTUs) present in blank control. Sequence counts were obtained using the biom summarize-table script.

\begin{tabular}{ccc}
\hline Sample & Counts Prior Filtering & Counts after Filtering \\
\hline Blank & 3824 & NA \\
FI3 & 78,903 & 78,881 \\
FI9 & 24,956 & 24,495 \\
FI12 & 56,971 & 56,846 \\
NASD3 & 32,086 & 14,768 \\
NASD14 & 44,255 & 24,740 \\
NASD22 & 60,266 & 55,727 \\
NASD27 & 24,633 & 13,392 \\
NASD29 & 101,517 & 100,028 \\
\hline
\end{tabular}


A.

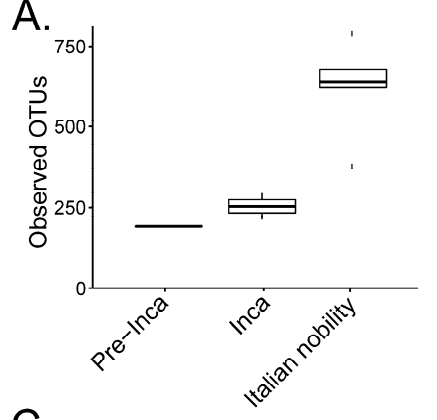

C.

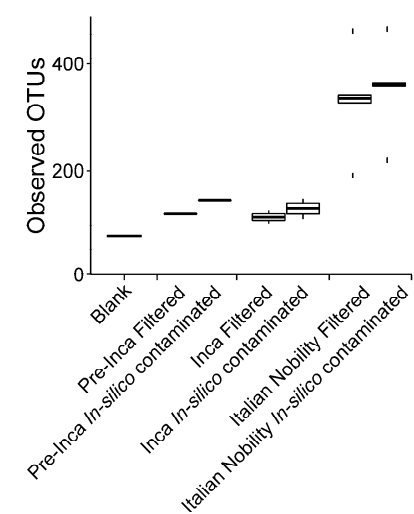

B.

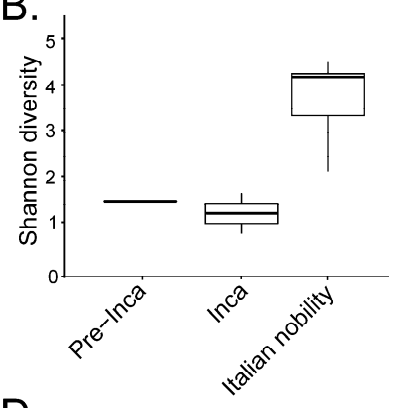

D.

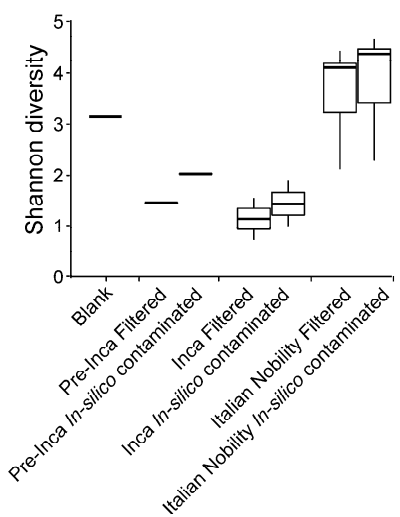

Figure 1. Alpha diversity of the mummified gut remains. Results show the alpha diversity values of the pre-Inca/Inca and Italian nobility mummies. Observed OTUs (A) and evenness (Shannon diversity index) (B) were calculated after filtering OTUs present in the blank control from the mummified microbiome (see text). Sequence files were also in-silico contaminated with the OTUs identified in the blank control to mimic cross contamination of the samples with extraction blanks. Observed OTUs (C) and evenness (Shannon diversity index) (D) were also determined for the in-silico contaminated samples.

A.

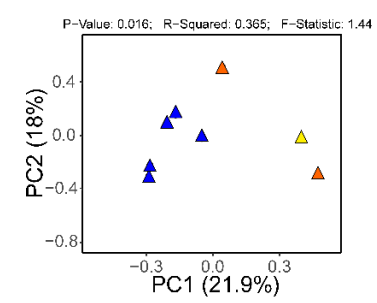

C.

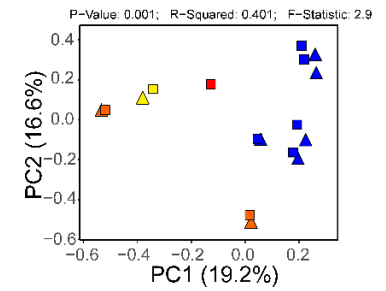

B.

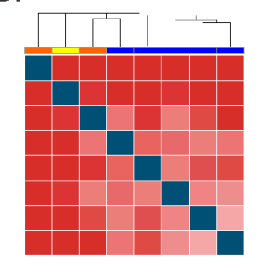

D.

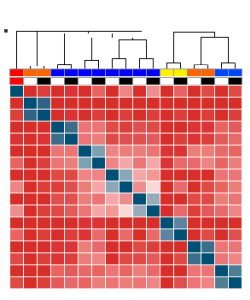

In-silico contaminated

- Yes

Blank

$\square$ Pre-Inca in-silico contaminated

$\triangle$ Pre-Inca filtered

Inca in-silico contaminated

nca filtered

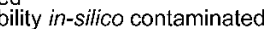

Italian nobility filtered

Figure 2. Beta diversity of the mummified gut remains. Results show the beta-diversity (Bray Curtis index) visualized as Principal Coordinate Analyses (PCoA) (A) and hierarchical cluster plots (B). Values were calculated after filtering OTUs present in the blank control from the mummified microbiome (see text). Results also show the beta-diversity (Bray-Curtis index) of the in-silico contaminated samples (see text) compared to the filtered samples. Results were visualized as PCoA plots (C) and hierarchical cluster plots (D). 
A.

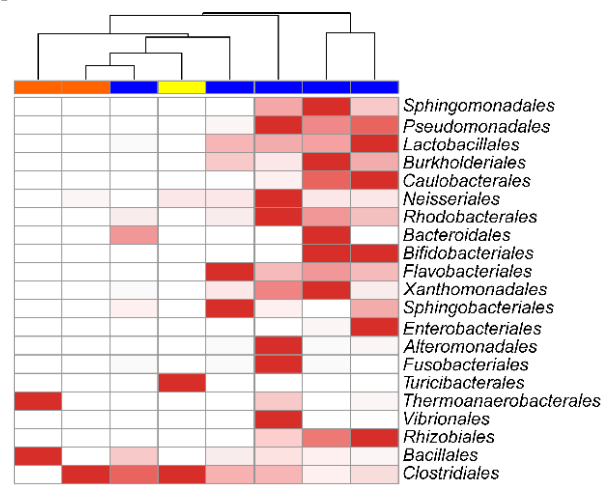

C.

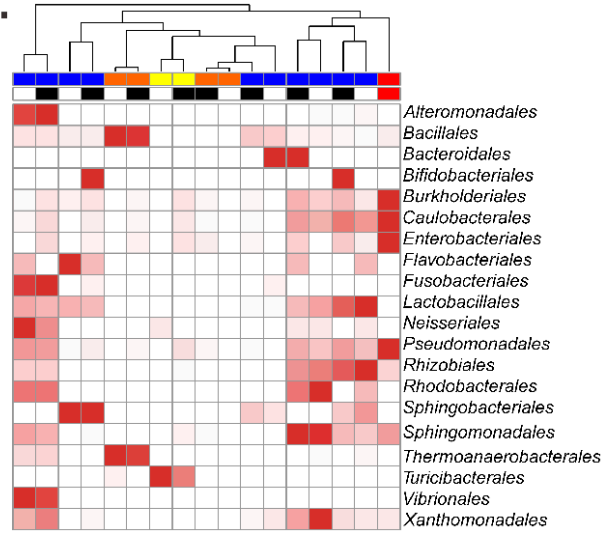

B.

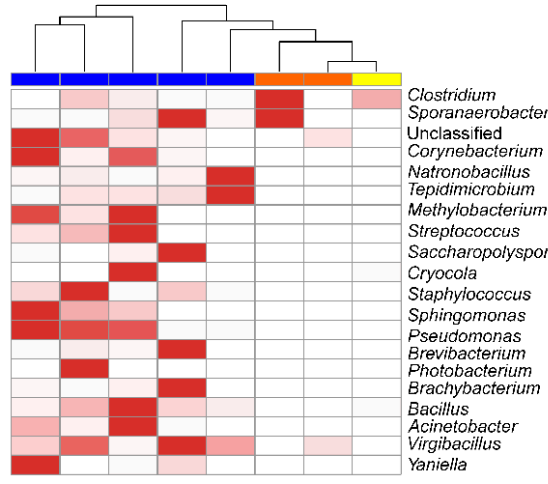

D.

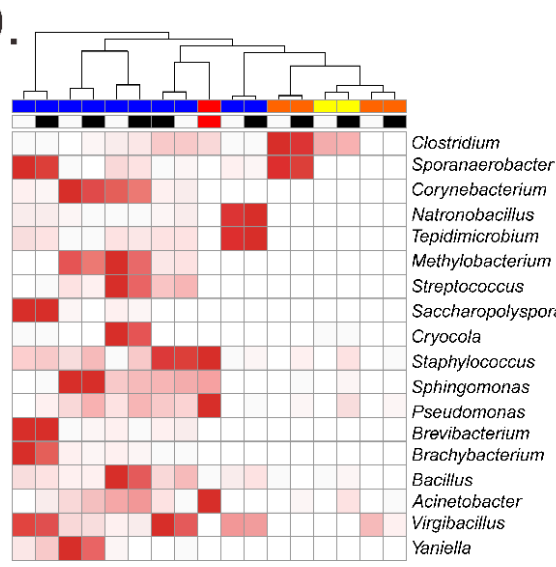

In-silico contaminated

No

Blank

Pre-Inca

Inca

Italian nobility

Figure 3. Heatmap of relative abundances of selected taxa. Results show the relative abundances of selected OTUs at the order (A) and genus level (B). Values were plotted after filtering OTUs present in the blank control from the mummified microbiome (see text). Results also show the relative abundances of selected OTUs at the order (C) and genus level (D) of the in-silico contaminated samples (see text) compared to the filtered samples. 
Results from the in-silico contamination seem to support that the patterns observed in the previous article are not due to contamination of the extraction blank, and that the separation may be likely due to the differences mentioned in the original article, including dietary habits [2]. The in-silico contamination showed that a blank control may not necessarily affect the patterns observed in microbiome samples, but that any changes in observed patterns may be due by the extent of the contamination of the blank control. In addition, under any circumstances, the possibility of contamination is intrinsic to ancient DNA and microbiome work. It would be impossible to determine if the "contamination event" occurred anciently or recently; however, external artifacts are more prone to either type of contamination; in our case, specifically, the samples were obtained as they would be under surgery, and in specialized ancient DNA laboratories.

\section{Considerations about Microbial Resilience in Ancient Samples}

Numerous mechanisms, other than contamination, can explain these results, including the nature of the samples, horizontal gene transfer, sporulation, dormancy, and stress response, just to mention a few [27]. When DNA damage occurs, again, mostly upon cell death, tools such as mapDamage can aid to assess misincorporations within a particular sequence [28]. We have repeatedly stated that mapDamage provides very useful information when only one genome is being considered. In addition, cytosine deamination may be strongly influenced by sample age and temperature of collection site [29]. Utilizing mapDamage in microbiomes would also require deep sequencing, which is often costly, to correctly assess misincorporations. In fact, mapDamage analyses would have to be performed in the hundreds of different species genomes present in a sample. Failing to find misincorporations in sequences present in microbiome samples that have failed to reach enough coverage is not necessarily evidence of contamination.

In terms of self-plagiarism mentioned by Eisenhofer and Weyrich, the mapDamage original results were published in FEMS Microbiology Letters [30]. The journal is currently part of the Oxford Academic journals, and as part of the copyright agreement with Oxford University Press, we have retained the right to use all or part of the article and abstract, including the supplementary figures after publication.

As we have previously done in numerous occasions, we thank our concerned colleagues for their comments. However, science may be better served by possible collaborations and publishing original research rather than after-the-fact criticism. As we obtain data on ancient DNA, we continue to better understand the microbiome of ancient samples, taking into consideration what we know so far about modern microbiomes, and the numerous mechanisms of microbial resilience and DNA repair. We will continue to report our findings on data obtained following strict guidelines to avoid contamination and will keep carrying out original research that will result in a better understanding of ancient ethnic groups despite possible shortcomings.

Author Contributions: T.S.M.-R. re-analyzed the data, prepared the figures and wrote the manuscript. G.A.T. wrote and revised the manuscript.

Funding: This study did not receive funding.

Conflicts of Interest: The authors declare no conflict of interest.

\section{References}

1. Eisenhofer, R.; Weyrich, L.S. Proper authentication of ancient DNA is still essential. Genes 2018, 9, 122. [CrossRef] [PubMed]

2. Santiago-Rodriguez, T.M.; Fornaciari, G.; Luciani, S.; Toranzos, G.A.; Marota, I.; Giuffra, V.; Cano, R.J. Gut microbiome and putative resistome of inca and italian nobility mummies. Genes 2017, 8, 310. [CrossRef] [PubMed] 
3. Bokulich, N.A.; Kaehler, B.D.; Rideout, J.R.; Dillon, M.; Bolyen, E.; Knight, R.; Huttley, G.A.; Caporaso, J.G. Optimizing taxonomic classification of marker-gene amplicon sequences with QIIME 2's q2-feature-classifier plugin. Microbiome 2018, 6, 90. [CrossRef] [PubMed]

4. Velsko, I.M.; Frantz, L.A.F.; Herbig, A.; Larson, G.; Warinner, C. Selection of appropriate metagenome taxonomic classifiers for ancient microbiome research. bioRxiv 2018, 260042. [CrossRef]

5. Menzel, P.; Ng, K.L.; Krogh, A. Fast and sensitive taxonomic classification for metagenomics with Kaiju. Nat. Commun. 2016, 7, 11257. [CrossRef] [PubMed]

6. D'Auria, G.; Peris-Bondia, F.; Džunková, M.; Mira, A.; Collado, M.C.; Latorre, A.; Moya, A. Active and secreted IgA-coated bacterial fractions from the human gut reveal an under-represented microbiota core. Sci. Rep. 2013, 3, 3515. [CrossRef] [PubMed]

7. Adler, C.J.; Dobney, K.; Weyrich, L.S.; Kaidonis, J.; Walker, A.W.; Haak, W.; Bradshaw, C.J.A.; Townsend, G.; Sołtysiak, A.; Alt, K.W.; et al. Sequencing ancient calcified dental plaque shows changes in oral microbiota with dietary shifts of the Neolithic and Industrial revolutions. Nat. Genet. 2013, 45, 450-455. [CrossRef] [PubMed]

8. Rascovan, N.; Huynh, H.; Chouin, G.; Adekola, K.; Georges-Zimmermann, P.; Signoli, M.; Desfosses, Y.; Aboudharam, G.; Drancourt, M.; Desnues, C. Tracing back ancient oral microbiomes and oral pathogens using dental pulps from ancient teeth. npj Biofilms Microbiomes 2016, 2, 6. [CrossRef] [PubMed]

9. Hallmaier-Wacker, L.K.; Lueert, S.; Roos, C.; Knauf, S. The impact of storage buffer, DNA extraction method, and polymerase on microbial analysis. Sci. Rep. 2018, 8, 6292. [CrossRef] [PubMed]

10. Edmonds, K.; Williams, L. The Role of the Negative Control in Microbiome Analyses. FASEB J. 2017, 31, $940-943$.

11. Kim, D.; Hofstaedter, C.E.; Zhao, C.; Mattei, L.; Tanes, C.; Clarke, E.; Lauder, A.; Sherrill-Mix, S.; Chehoud, C.; Kelsen, J.; et al. Optimizing methods and dodging pitfalls in microbiome research. Microbiome 2017, 5, 52. [CrossRef] [PubMed]

12. Plaza-Díaz, J.; Fernández-Caballero, J.Á.; Chueca, N.; García, F.; Gómez-Llorente, C.; Sáez-Lara, M.J.; Fontana, L.; Gil, Á. Pyrosequencing analysis reveals changes in intestinal microbiota of healthy adults who received a daily dose of immunomodulatory probiotic strains. Nutrients 2015, 7, 3999-4015. [CrossRef] [PubMed]

13. Pérez-Santiago, J.; Gianella, S.; Massanella, M.; Spina, C.A.; Karris, M.Y.; Var, S.R.; Patel, D.; Jordan, P.S.; Young, J.A.; Little, S.J.; et al. Gut Lactobacillales are associated with higher CD4 and less microbial translocation during HIV infection. AIDS 2013, 27, 1921-1931. [CrossRef] [PubMed]

14. Gupta, S.; Mohammed, M.; Ghosh, T.; Kanungo, S.; Nair, G.; Mande, S.S. Metagenome of the gut of a malnourished child. Gut Pathog. 2011, 3, 7. [CrossRef] [PubMed]

15. Peris-Bondia, F.; Latorre, A.; Artacho, A.; Moya, A.; D'Auria, G. The active human gut microbiota differs from the total microbiota. PLoS ONE 2011, 6, e22448. [CrossRef] [PubMed]

16. Sommer, M.O.A.; Church, G.M.; Dantas, G. The human microbiome harbors a diverse reservoir of antibiotic resistance genes. Virulence 2010, 1, 299-303. [CrossRef] [PubMed]

17. Allali, I.; Delgado, S.; Marron, P.I.; Astudillo, A.; Yeh, J.J.; Ghazal, H.; Amzazi, S.; Keku, T.; Azcarate-Peril, M.A. Gut microbiome compositional and functional differences between tumor and non-tumor adjacent tissues from cohorts from the US and Spain. Gut Microbes 2015, 6, 161-172. [CrossRef] [PubMed]

18. Chen, Y.; Ji, F.; Guo, J.; Shi, D.; Fang, D.; Li, L. Dysbiosis of small intestinal microbiota in liver cirrhosis and its association with etiology. Sci. Rep. 2016, 6, 34055. [CrossRef] [PubMed]

19. Bashiardes, S.; Shapiro, H.; Rozin, S.; Shibolet, O.; Elinav, E. Non-alcoholic fatty liver and the gut microbiota. Mol. Metab. 2016, 5, 782-794. [CrossRef] [PubMed]

20. Stearns, J.C.; Lynch, M.D.J.; Senadheera, D.B.; Tenenbaum, H.C.; Goldberg, M.B.; Cvitkovitch, D.G.; Croitoru, K.; Moreno-Hagelsieb, G.; Neufeld, J.D. Bacterial biogeography of the human digestive tract. Sci. Rep. 2011, 1, 170. [CrossRef] [PubMed]

21. Rajilić-Stojanović, M.; de Vos, W.M. The first 1000 cultured species of the human gastrointestinal microbiota. FEMS Microbiol. Rev. 2014, 38, 996-1047. [CrossRef] [PubMed]

22. Bull, M.J.; Plummer, N.T. Part 1: The Human Gut Microbiome in Health and Disease. Integr. Med. 2014, 13, 17-22.

23. Struzycka, I. The oral microbiome in dental caries. Pol. J. Microbiol. 2014, 63, 127-135. [PubMed] 
24. The Human Microbiome Project Consortium; Huttenhower, C.; Gevers, D.; Knight, R.; Abubucker, S.; Badger, J.H.; Chinwalla, A.T.; Creasy, H.H.; Earl, A.M.; FitzGerald, M.G.; et al. Structure, function and diversity of the healthy human microbiome. Nature 2012, 486, 207-214. [CrossRef]

25. Hartman, A.L.; Lough, D.M.; Barupal, D.K.; Fiehn, O.; Fishbein, T.; Zasloff, M.; Eisen, J.A. Human gut microbiome adopts an alternative state following small bowel transplantation. Proc. Natl. Acad. Sci. USA 2009, 106, 17187-17192. [CrossRef] [PubMed]

26. Kuczynski, J.; Stombaugh, J.; Walters, W.A.; González, A.; Caporaso, J.G.; Knight, R. Using QIIME to analyze 16s rRNA gene sequences from microbial communities. Curr. Protoc. Microbiol. 2012, 27. [CrossRef]

27. MacKelprang, R.; Burkert, A.; Haw, M.; Mahendrarajah, T.; Conaway, C.H.; Douglas, T.A.; Waldrop, M.P. Microbial survival strategies in ancient permafrost: Insights from metagenomics. ISME J. 2017, 11, 2305-2318. [CrossRef] [PubMed]

28. Jónsson, H.; Ginolhac, A.; Schubert, M. mapDamage2.0: fast approximate Bayesian estimates of ancient DNA damage parameters. Bioinformatics 2013, 29, 1682-1684. [CrossRef] [PubMed]

29. Kistler, L.; Ware, R.; Smith, O.; Collins, M.; Allaby, R.G. A new model for ancient DNA decay based on paleogenomic meta-analysis. Nucleic Acids Res. 2017, 45, 6310-6320. [CrossRef] [PubMed]

30. Santiago-Rodriguez, T.M.; Fornaciari, G.; Luciani, S.; Dowd, S.E.; Toranzos, G.A.; Marota, I.; Cano, R.J. Natural mummification of the human gut preserves bacteriophage DNA. FEMS Microbiol. Lett. 2015, 363. [CrossRef] [PubMed]

(C) 2018 by the authors. Licensee MDPI, Basel, Switzerland. This article is an open access article distributed under the terms and conditions of the Creative Commons Attribution (CC BY) license (http://creativecommons.org/licenses/by/4.0/). 